\title{
Impact of percutaneous valve intervention on mechanical valves selection
}

\author{
Khalid $\mathrm{Al}_{e}$ brahim ${ }^{1}$ \\ ${ }^{1}$ King Abdulaziz University Hospital
}

January 27, 2022

Letter to the editor

Impact of percutaneous valve intervention on mechanical valves selection Khaled Ebrahim Al-Ebrahim MBBCh, FRCSC

Department of surgery, King Abdulaziz University Hospital, King Abdulaziz University Jeddah, Saudi Arabia

Corresponding Author

Khaled Ebrahim Al-Ebrahim, King Abdulaziz University Hospital, Jeddah, Saudi Arabia.

Tel.966 559625170

Email: dr.k.ebrahim@gmail.com

To the Editor

I congratulate Shojaeifard and colleagues for their successful management of mechanical tricuspid valve thrombosis ${ }^{1}$. Cardiac surgery nowadays is rapidly changing and at a crucial turning point. Given the swift advances in interventional cardiology, cardiac surgery operative decisions and strategy is different from the past when we started cardiac surgery. The selection challenges between mechanical and bio prothesis choice is now clearer and more obvious. Valve repair is always the gold standard in any valve procedure as replacement of either valve type will carry its own morbidity and mortality and eventually, we are replacing one disease with another. From our experience in the third world countries, the old concept that mechanical valves are cost effective and better on the long run because of its durability and avoidance of reoperation proved to be wrong. In contrary to bio prostheses, mechanical valves entail close and strict follow up of anticoagulation with all its thromboembolic and hemorrhagic complications of this "rat poison" . Tricuspid valve replacement especially when using mechanical valves is considered by most surgeons as a catastrophic procedure $^{2}$. Recently, the advent of trans catheter procedures on aortic, mitral, and tricuspid vale, repair and implantation really opened the door widely for the interventional cardiologist to play a major role in all valve procedures and affecting the volume and future of cardiac surgery practice and resident training ${ }^{3}$. Transcatheter valve-in-valve implantation within dysfunctional surgical bioprosthesis has become an alternative to high risk redo open-heart surgery ${ }^{4}$. This will change dramatically the trade- offs between mechanical and bioprosthetic valve selection and may affect the future of mechanical valves.

References

Shojaeifard M, Omidi N, Erami S,et al. Mechanical tricuspid valve thrombosis: a mid term follow-up study.JCardSurg. 2022;1-10.doi:10.1111/jocs.16209 
Sung K, Park PW, Park KH, Jun TG, Lee YT, Yang JH, Kim WS, Hwang J. Is tricuspid valve replacement a catastrophic operation? Eur J Cardiothorac Surg. 2009 Nov;36(5):825-9. doi: 10.1016/j.ejcts.2009.04.063. Epub 2009 Jul 8. PMID: 19589692.

3-Al-Ebrahim EK, Madani TA, Al-Ebrahim KE. Future of cardiac surgery, introducing the interventional surgeon. Journal of Cardiac Surgery. 2022 Jan;37(1):88-92. 4- Deshpande SP, Sankova S, Dorsey N, Dawood MY, Tanaka K. Transcatheter tricuspid valve-in valve replacement-hope for the forgotten valve! Korean J Anesthesiol. 2020 Jun;73(3):264-266. doi: 10.4097/kja.20104. Epub 2020 Mar 12. PMID: 32160737; PMCID: PMC7280897. 